\title{
Characterizing the Etiologies of Seizures and Cortical Myoclonic Activity in Covid-19 Patients and their Impact on Outcomes
}

\author{
Bhasin $A^{1 \dagger}$, Kappagantu $A^{1 \dagger}$, Brantz $\mathrm{HA}^{1 \dagger}$, Ali $\mathbf{R}^{1 \dagger}$, Jageka $\mathrm{CJ}^{1 \dagger}$, Stopa $\mathrm{BM}^{2,3}$, Izzy $\mathrm{S}^{4}$ and Khawaja $\mathrm{AM}^{5^{\star}}$ \\ ${ }^{1}$ Wayne State University School of Medicine, Detroit, MI, USA \\ ${ }^{2}$ Computational Neuroscience Outcomes Center at Harvard, Brigham and Women's Hospital, Boston, MA, USA \\ ${ }^{3}$ Virginia Tech Carilion School of Medicine, Roanoke, VA, USA \\ ${ }^{4}$ Department of Neurology, Division of Neurointensive care and Cerebrovascular diseases, Brigham and Women's Hospital, Boston, \\ MA, USA \\ ${ }^{5}$ Department of Neurology, Division of Neurointensive care and Cerebrovascular diseases, Wayne State University, Detroit, MI, USA
}

†These authors contributed equally to the manuscript

${ }^{*}$ Corresponding author: Khawaja AM, MD. 4201 St. Antoine, UHC-8D, Detroit, MI 48201, USA, Tel: +1-313-745-2892, E-mail: akhawaja@wayne.edu

Citation: Bhasin A, Kappagantu A, Brantz HA, Ali R, Jageka C, et al. (2022) Characterizing the Etiologies of Seizures and Cortical Myoclonic Activity in Covid-19 Patients and their Impact on Outcomes. J Neurol Neurol Disord 8(1): 101

\begin{abstract}
Objective: To characterize underlying etiologies, management and outcomes of seizures, status epilepticus, and cortical myoclonic activity (CMA) in COVID-19, with individual patient data analysis of published literature.
\end{abstract}

Methods: Systematic literature review was conducted. Criteria included seizures, status epilepticus, and/or CMA developing prior to or during hospitalization, with concomitant COVID-19. COVID-19 severity was dichotomized into mild and severe cases, based on severity of respiratory symptoms. Good outcome was defined as discharge without severe deficits and/or return to near baseline.

Results: A total of 105 studies reporting 176 patients (male 56.3\%; mean age 47.8, SD 25.6) were included. Status epilepticus occurred in 47 patients (26.7\%), CMA in 41 (23.3\%), and Severe COVID-19 in 90 (53.6\%). Seizure-like activity was noted on electroencephalography in 52/103 patients (50.5\%). Abnormal cerebrospinal fluid analysis was reported in 40/92 (43.5\%) patients. Most common underlying diagnosis amongst known etiology was encephalitis in 47/91 patients (51.6\%), followed by infarct $(n=18 ; 19.8 \%)$ and intracranial hemorrhage $(n=14 ; 15.4 \%)$. The most common antiepileptic medication was levetiracetam (93/130; 71.5\%). Pulse-dose steroids were used in 32 (19.3\%) patients, whereas intravenous immmunoglobulin was used in $22(13.4 \%)$. Overall, 101 patients (63.9\%) had good outcomes while 24 (14.6\%) died. In multivariate regression, severe COVID-19 (OR=0.116), age $(\mathrm{OR}=0.980)$ and intubation $(\mathrm{OR}=0.303)$ were associated with worse outcomes. In a separate regression model, encephalitis was independently associated with good outcomes $(\mathrm{OR}=6.07)$, whereas severe COVID-19 predicted worse outcomes $(\mathrm{OR}=0.17)$.

Significance: Overall outcomes were good in most patients, indicating efficacy of existing antiepileptic treatments. Despite severe COVID-19 strongly predicting poor outcomes, identification of encephalitis as an underlying etiology was still 
independently associated with good outcomes. We recommend obtaining comprehensive neurologic workup to evaluate underlying etiology to direct targeted treatments to achieve better outcomes. Further research should focus on elucidating pathophysiology of encephalitis in COVID-19, and investigate long-term seizure and neurocognitive outcomes.

Keywords: Seizures; Status Epilepticus; Covid-19; Sars-Cov-2; Systematic Review; Outcomes; Encephalitis 


\section{Introduction}

COVID-19 has had a devastating impact across the world since the end of 2019. As of November 22 ${ }^{\text {nd }}$, 2021, the World Health Organization has reported over 256 million confirmed cases and over 5.1 million deaths globally [1]. A variety of neurologic conditions secondary to COVID-19 have been extensively published. In the literature covering adult patients, several case reports and series have been published covering description of seizures and cortical or sub-cortical myoclonus, electroencephalography (EEG) features, and cerebrospinal fluid (CSF) and neuroimaging findings [2-6]. There have also been reports of detection of COVID-19 antibodies or isolation of viral nucleic acid from the CSF, and association with COVID-19 related encephalitis [7,8]. Based on largescale studies, seizure incidence has ranged from 0.5-1.6\% in COVID-19 patients [9-11]. Yet, other studies have not detected any seizures, making this a rare event [12]. In the pediatric literature, these are reported to be even more rare [5,13]. Due to the significant variability in reporting and limitation to small-scale studies, the underlying etiologies of seizures and cortical myoclonic activity (CMA) in COVID-19, their treatment responsiveness, and impact on outcomes, are unknown. In addition, overall outcomes of these patients are also unknown. The purpose of this study is to address these knowledge gaps by aggregating data from various published clinical studies, in order to identify etiologies that could guide patient management in the rare occurrence of seizures and CMA in COVID-19, and investigate the impact of targeted treatments on outcomes.

\section{Methods}

\section{Search strategy and selection criteria}

The protocol for this systematic review has been published (PROSPERO: CRD42020202703) and was conducted in accordance with the PRISMA guidelines [14]. The Journal of the Medical Library Association's systematic approach to develop literature searches was used to design the search strategy for this systematic review [15]. Three independent investigators searched Web of Science, Scopus, PubMed, Google Scholar, and EMBASE databases for articles published prior to September $20^{\text {th }}, 2021$ using the following keywords: COVID-19, 2019-nCoV, SARS-CoV-2, neurologic manifestations, neurology, nervous system diseases, neurological complications, stroke, seizure, encephalopathy, CNS manifestations, neurologic symptoms, neurologic sequelae, and neurologic syndromes. From these search terms a formal search string was created and translated for the specific syntax required by each individual database. A total of 2,711 articles were screened, of which 556 articles underwent full text review (Figure 1).

Selected articles were included if they discussed individual patient-level data as a case series, retrospective or prospective cohort, or case report. All selected studies also mentioned at least one COVID-19 positive patient who had seizures or cortical myoclonic activity with concomitant COVID-19. Articles were excluded from this study if the full-length articles could not be retrieved, if they were review articles, or if they did not provide at least some individual patient-level data. Studies were also excluded if the patients discussed in the articles were not confirmed to be COVID-19 positive, or if the patients had neurologic manifestations other than seizures or CMA. The quality of each article was rated using the National Institutes of Health (NIH) Quality Assessment Tool for Systematic Reviews and Meta-Analyses [16].

Given these criteria, 451 of the 556 qualifying full-text articles that were individually-reviewed were excluded from the study, and were checked by a separate investigator who also resolved any disputes. Data from a total of 105 studies were used in this systematic review, including 69 case reports and 36 case series (Figure 1).

\section{Data extraction and definitions}

The following variables were collected for every patient in each study who tested positive for COVID-19 and developed seizures, status epilepticus, or CMA upon presentation and/or during their hospital stay: COVID-19 severity, Glasgow Coma Scale (GCS), length of hospital stay (LOS), age, sex, comorbidities, computed tomograpy (CT) and magnetic resonance imaging (MRI) imaging 
impressions, EEG findings, underlying acute or chronic neurologic conditions, name and number of anti-epileptic drugs used, pulse-dose steroids, intravenous immunoglobulin (IVIG), plasmapheresis (PLEX), use of specific COVID-19 targeted treatments, intubation status, and mortality.

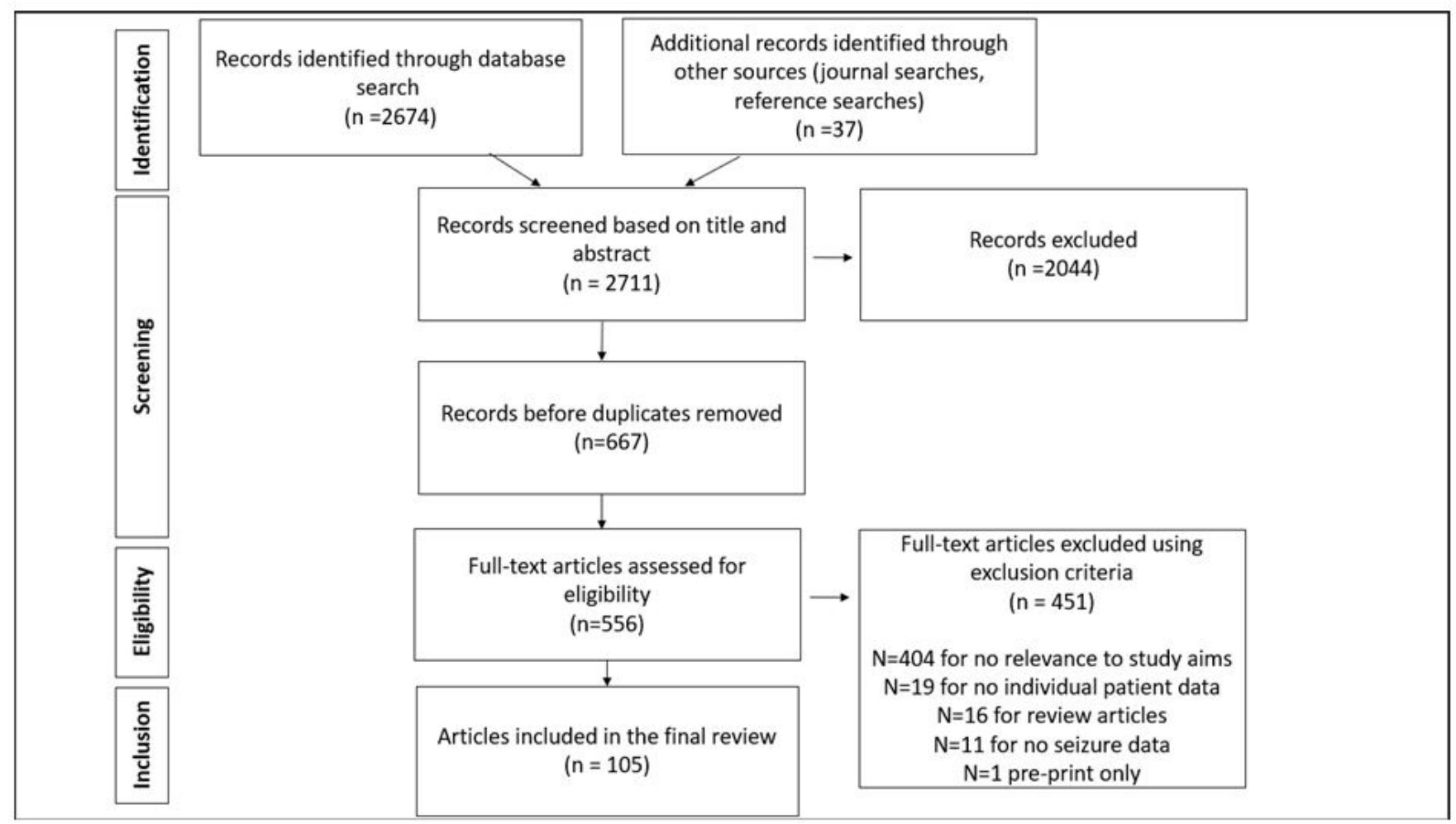

Figure 1: PRISMA Flowchart Screening and selection of studies utilizing a pre-specified search criteria applied to multiple libraries

A good outcome was designated when patients returned to normal or baseline without new severely-disabling deficits, and/or did not have a skilled nursing facility discharge designation. Severe COVID-19 was defined as hypoxia with dyspnea or tachypnea, and/ or the need for mechanical ventilation not from seizures or status epilepticus, or concomitant presence of multi-organ dysfunction with or without septic shock. If the severity status was provided by the authors, it was recorded as such. Abnormal cerebrospinal fluid (CSF) was defined by abnormally elevated protein above $80 \mathrm{mg} / \mathrm{dL}$, elevated white blood cell count above 5/microliter, elevated lactic acid, or abnormal CSF culture. Any EEG abnormality was defined as presence of any electrographic seizures, electrographic status epilepticus, or seizure-like abnormalities such as epileptiform discharges, periodic lateralized epileptiform discharges, and generalized periodic discharges. Seizures had to be either new-onset, or recurrent with pre-existing epilepsy only if the epilepsy was well-controlled at baseline as specifically mentioned by study authors. Status epilepticus was defined as continuous seizures for at least 5 minutes duration or recurrent seizures without return to baseline, or presence of multiple electrographic seizures or a persistent interictal EEG pattern with or without clinical manifestations, or designated if the authors had explicitly classified seizures as status epilepticus. CMA was included if generalized and severe in nature, accompanied by significant mental status changes, having associated electrographic seizure or seizure-like features, have a potential epileptogenic nidus in cerebral cortex on neuroimaging, or show improvement with antiepileptics. Underlying neurologic diagnosis included any neurologic condition concomitant to seizures or CMA, whether reported by authors or inferred from investigations.

\section{Data synthesis and statistical analysis}

Categorical variables were presented as percentage, and continuous variables as mean and standard deviation (SD) or median and interquartile range (IQR). Meta-analysis was not conducted due to the relative dearth of high-quality large-scale cohort studies. 
Variables were compared using chi-squared or student's t-test statistics to assess uni-variate assoications. The association between outcome and predicting variables (excluding seizures without status epilepticus) was studied in a multivariate binary logistic regression model. Model construction was based on various factors including number of missing observations (e.g. GCS, COVID-19 targeted treatments), clinically-determined covariate interactions, and model fit determined by Pseudo $\mathrm{R}^{2}$ metric. The association of outcomes with encephalitis being the main explanatory variable was assessed in separate regression models. Finally, additional regression models were constructed to assess the association between status epilepticus and CMA with various predictors. All analyses were completed using STATA v.13 (College Station, TX). A p-value at $<0.05$ was considered statistically significant.

\section{Results}

A total of 105 studies ( $\mathrm{n}=176$ patients) were included in the final analysis, with case series comprising 36 studies (Figure 1). All studies were of fair or good quality as assessed by the National Institutes of Health (NIH) Quality Assessment Tool. A total of 156 (88.6\%) patients with clinical or electrographic seizures were reported. Status epilepticus occurred in 47 (26.7\%) patients. Cortical Myoclonic Activity (CMA) occurred in 41 (23.3\%) patients, of which 21 had both CMA and seizure (Table 1). The majority of seizure presentation was new-onset $(93.8 \%)$.

\begin{tabular}{|c|c|c|c|c|c|c|c|c|c|c|c|}
\hline Variable & $\begin{array}{c}\text { All } \\
\text { patients } \\
(n=176)\end{array}$ & $\begin{array}{c}\text { Number } \\
\text { data } \\
\text { available }\end{array}$ & $\begin{array}{c}\text { Non- } \\
\text { severe } \\
\text { COVID- } \\
19 \\
(n=90)\end{array}$ & $\begin{array}{c}\text { Severe } \\
\text { COVID- } \\
19 \\
(n=78)\end{array}$ & $\begin{array}{c}\text { p- } \\
\text { value }\end{array}$ & $\begin{array}{c}\text { No status } \\
\text { epilepticus } \\
(n=126)\end{array}$ & $\begin{array}{c}\text { Status } \\
\text { epilepticus } \\
(n=50)\end{array}$ & $\begin{array}{c}\text { p- } \\
\text { value }\end{array}$ & $\begin{array}{c}\text { No CMA } \\
(n=134)\end{array}$ & $\begin{array}{c}\text { CMA } \\
(n=42)\end{array}$ & p-value \\
\hline $\begin{array}{c}\text { Age, mean } \\
(\mathrm{SD})\end{array}$ & $\begin{array}{c}47.8 \\
(25.6) \\
\end{array}$ & $\begin{array}{c}176 \\
(100 \%) \\
\end{array}$ & $\begin{array}{c}42.2 \\
(26.7) \\
\end{array}$ & $\begin{array}{r}52.3 \\
(23.5) \\
\end{array}$ & 0.011 & $48.9(24.8)$ & $45.0(27.6)$ & 0.367 & $\begin{array}{c}43.2 \\
(26.5) \\
\end{array}$ & $\begin{array}{c}62.2 \\
(15.0) \\
\end{array}$ & $<0.001$ \\
\hline $\begin{array}{c}\text { Male sex, } \mathrm{n} \\
(\%)\end{array}$ & $\begin{array}{c}99 \\
(56.3 \%)\end{array}$ & $\begin{array}{c}176 \\
(100 \%) \\
\end{array}$ & $\begin{array}{c}49 \\
(54.4 \%) \\
\end{array}$ & $\begin{array}{c}44 \\
(56.4 \%) \\
\end{array}$ & 0.798 & $76(60.3 \%)$ & $23(46.0 \%)$ & 0.084 & $\begin{array}{c}69 \\
(51.5 \%) \\
\end{array}$ & $\begin{array}{c}30 \\
(71.4 \%) \\
\end{array}$ & 0.023 \\
\hline $\begin{array}{c}\text { Glasgow } \\
\text { coma scale, } \\
\text { mean }(\mathrm{SD})\end{array}$ & $\begin{array}{l}10.4 \\
(4.3)\end{array}$ & $\begin{array}{c}51 \\
(29.0 \%)\end{array}$ & $12.0(3.9)$ & $8.1(3.9)$ & 0.001 & $10.9(4.3)$ & $8.9(4.2)$ & 0.146 & $10.7(4.2)$ & $9.7(4.6)$ & 0.462 \\
\hline $\begin{array}{c}\text { Length of } \\
\text { stay, mean } \\
\text { (SD) }\end{array}$ & $\begin{array}{c}22.1 \\
(24.4)\end{array}$ & $\begin{array}{c}90 \\
(51.1 \%)\end{array}$ & $\begin{array}{c}13.9 \\
(13.2)\end{array}$ & $\begin{array}{c}30.0 \\
(29.7)\end{array}$ & 0.001 & $22.8(26.0)$ & $20.8(21.3)$ & 0.707 & $\begin{array}{c}18.8 \\
(22.5)\end{array}$ & $\begin{array}{l}33.1 \\
(6.0)\end{array}$ & 0.018 \\
\hline $\begin{array}{c}\text { Status } \\
\text { epilepticus, } \\
\text { n (\%) }\end{array}$ & $\begin{array}{c}50 \\
(28.4 \%)\end{array}$ & $\begin{array}{c}176 \\
(100 \%)\end{array}$ & $\begin{array}{c}26 \\
(28.9 \%)\end{array}$ & $\begin{array}{c}23 \\
(29.5 \%)\end{array}$ & 0.932 & & & & $\begin{array}{c}45 \\
(33.6 \%)\end{array}$ & $\begin{array}{c}5 \\
(11.9 \%)\end{array}$ & 0.007 \\
\hline $\begin{array}{c}\text { Severe } \\
\text { COVID-19, } \\
\text { n (\%) }\end{array}$ & $\begin{array}{c}78 \\
(46.4 \%)\end{array}$ & $\begin{array}{c}168 \\
(95.5 \%)\end{array}$ & & & & $55(46.2 \%)$ & $23(46.9 \%)$ & 0.932 & $\begin{array}{c}53 \\
(40.5 \%)\end{array}$ & $\begin{array}{c}25 \\
(67.6 \%)\end{array}$ & 0.004 \\
\hline $\begin{array}{c}\text { Cortical } \\
\text { myoclonic } \\
\text { activity, n } \\
(\%)\end{array}$ & $\begin{array}{c}42 \\
(23.9 \%)\end{array}$ & $\begin{array}{c}176 \\
(100 \%)\end{array}$ & $\begin{array}{c}12 \\
(13.3 \%)\end{array}$ & $\begin{array}{c}25 \\
(32.1 \%)\end{array}$ & 0.004 & 37 (29.4\%) & $5(10.0 \%)$ & 0.007 & & & \\
\hline $\begin{array}{c}\text { Any seizure- } \\
\text { like EEG } \\
\text { abnormality, } \\
\text { n (\%) }\end{array}$ & $\begin{array}{c}52 \\
(50.5 \%)\end{array}$ & $\begin{array}{c}103 \\
(58.5 \%)\end{array}$ & $\begin{array}{c}21 \\
(42.9 \%)\end{array}$ & $\begin{array}{c}29 \\
(55.8 \%)\end{array}$ & 0.195 & $28(42.4 \%)$ & $24(64.9 \%)$ & 0.029 & $\begin{array}{c}43 \\
(56.6 \%)\end{array}$ & $\begin{array}{c}9 \\
(33.3 \%)\end{array}$ & 0.038 \\
\hline $\begin{array}{l}\text { Abnormal } \\
\text { CSF, n (\%) }\end{array}$ & $\begin{array}{c}40 \\
(43.5 \%)\end{array}$ & $\begin{array}{c}92 \\
(52.3 \%)\end{array}$ & $\begin{array}{c}23 \\
(47.9 \%)\end{array}$ & $\begin{array}{c}15 \\
(39.5 \%)\end{array}$ & 0.377 & 25 (39.7\%) & $15(51.7 \%)$ & 0.279 & $\begin{array}{c}28 \\
(43.8 \%) \\
\end{array}$ & $\begin{array}{c}12 \\
(42.9 \%)\end{array}$ & 0.937 \\
\hline $\begin{array}{c}\text { Intubation, } \\
\mathrm{n}(\%)\end{array}$ & $\begin{array}{c}74 \\
(51.0 \%)\end{array}$ & $\begin{array}{c}145 \\
(82.4 \%)\end{array}$ & $\begin{array}{c}19 \\
(23.8 \%)\end{array}$ & $\begin{array}{c}55 \\
(84.6 \%)\end{array}$ & $<0.001$ & $43(42.2 \%)$ & $31(72.1 \%)$ & 0.001 & $\begin{array}{c}55 \\
(47.0 \%)\end{array}$ & $\begin{array}{c}19 \\
(67.9 \%)\end{array}$ & 0.047 \\
\hline
\end{tabular}




\begin{tabular}{|c|c|c|c|c|c|c|c|c|c|c|c|}
\hline $\begin{array}{c}\text { Mass lesion, } \\
\mathrm{n}(\%)\end{array}$ & $\begin{array}{c}42 \\
(46.2 \%) \\
\end{array}$ & $\begin{array}{c}93 \\
(52.8 \%) \\
\end{array}$ & $\begin{array}{c}18 \\
(39.1 \%) \\
\end{array}$ & $\begin{array}{c}19 \\
(47.5 \%) \\
\end{array}$ & 0.434 & 27 (42.9\%) & $15(53.6 \%)$ & 0.344 & $\begin{array}{c}37 \\
(52.1 \%) \\
\end{array}$ & $\begin{array}{c}5 \\
(25.0 \%) \\
\end{array}$ & 0.032 \\
\hline Encephalitis & $\begin{array}{c}47 \\
(50.5 \%) \\
\end{array}$ & $\begin{array}{c}93 \\
(52.8 \%) \\
\end{array}$ & $\begin{array}{c}28 \\
(60.9 \%) \\
\end{array}$ & $\begin{array}{c}17 \\
(42.5 \%) \\
\end{array}$ & 0.089 & $33(50.8 \%)$ & $14(50.0 \%)$ & 0.946 & $\begin{array}{c}33 \\
(46.5 \%) \\
\end{array}$ & $\begin{array}{c}14 \\
(63.6 \%) \\
\end{array}$ & 0.160 \\
\hline $\begin{array}{c}\text { Mortality, n } \\
(\%)\end{array}$ & $\begin{array}{c}24 \\
(14.6 \%) \\
\end{array}$ & $\begin{array}{c}165 \\
(93.8 \%) \\
\end{array}$ & $3(3.4 \%)$ & $\begin{array}{c}21 \\
(27.6 \%) \\
\end{array}$ & $<0.001$ & $13(11.2 \%)$ & $11(22.5 \%)$ & 0.061 & $\begin{array}{c}20 \\
(15.6 \%) \\
\end{array}$ & $\begin{array}{c}4 \\
(10.8 \%) \\
\end{array}$ & 0.464 \\
\hline $\begin{array}{c}\text { Good } \\
\text { outcome, n } \\
(\%)\end{array}$ & $\begin{array}{c}101 \\
(63.9 \%)\end{array}$ & $\begin{array}{c}158 \\
(89.8 \%)\end{array}$ & $\begin{array}{c}79 \\
(88.8 \%)\end{array}$ & $\begin{array}{c}22 \\
(31.9 \%)\end{array}$ & $<0.001$ & 74 (67.9\%) & $27(55.1 \%)$ & 0.122 & $\begin{array}{c}83 \\
(66.9 \%)\end{array}$ & $\begin{array}{c}18 \\
(52.9 \%)\end{array}$ & 0.132 \\
\hline $\begin{array}{c}\text { AED } \\
\text { number, } \\
\text { mean }(\mathrm{SD})\end{array}$ & $\begin{array}{c}1.4 \\
(0.99)\end{array}$ & $\begin{array}{c}133 \\
(75.6 \%)\end{array}$ & $1.4(1.0)$ & $1.5(1.0)$ & 0.559 & $1.1(0.8)$ & $2.0(1.2)$ & $<0.001$ & $1.4(0.9)$ & $\begin{array}{c}1.3 \\
(1.2)\end{array}$ & 0.640 \\
\hline $\begin{array}{c}\text { Pulse } \\
\text { steroids } \\
\mathrm{n}(\%)\end{array}$ & $\begin{array}{c}32 \\
(19.3 \%)\end{array}$ & $\begin{array}{c}166 \\
(94.3 \%)\end{array}$ & $\begin{array}{c}16 \\
(18.8 \%)\end{array}$ & $\begin{array}{c}17 \\
(22.7 \%)\end{array}$ & 0.549 & $21(17.7 \%)$ & $12(25.5 \%)$ & 0.251 & $\begin{array}{c}22 \\
(17.7 \%)\end{array}$ & $\begin{array}{c}11 \\
(26.2 \%)\end{array}$ & 0.236 \\
\hline $\begin{array}{c}\text { Any } \\
\text { steroid } \\
\text { dose, } \mathrm{n}(\%)\end{array}$ & $\begin{array}{c}41 \\
(34.5 \%)\end{array}$ & $\begin{array}{c}119 \\
(67.6 \%)\end{array}$ & $\begin{array}{c}17 \\
(24.3 \%)\end{array}$ & $\begin{array}{c}24 \\
(49.0 \%)\end{array}$ & 0.005 & $26(28.5 \%)$ & $15(53.6 \%)$ & 0.015 & $\begin{array}{c}26 \\
(28.0 \%)\end{array}$ & $\begin{array}{c}15 \\
(57.7 \%)\end{array}$ & 0.005 \\
\hline IVIG n(\%) & $\begin{array}{c}22 \\
(13.5 \%) \\
\end{array}$ & $\begin{array}{c}166 \\
(94.3 \%) \\
\end{array}$ & $\begin{array}{c}10 \\
(11.8 \%) \\
\end{array}$ & $\begin{array}{c}12 \\
(16.0 \%) \\
\end{array}$ & 0.438 & $13(10.9 \%)$ & $9(19.2 \%)$ & 0.159 & $\begin{array}{c}13 \\
(10.5 \%) \\
\end{array}$ & $\begin{array}{c}9 \\
(21.4 \%) \\
\end{array}$ & 0.071 \\
\hline PLEX & $4(2.4 \%)$ & $\begin{array}{c}160 \\
(90.9 \%)\end{array}$ & $1(1.2 \%)$ & $3(4.0 \%)$ & 0.254 & $3(2.5 \%)$ & $1(2.1 \%)$ & 0.882 & $2(1.6 \%)$ & $\begin{array}{c}2 \\
(4.8 \%)\end{array}$ & 0.25 \\
\hline
\end{tabular}

CMA: Cortical myoclonic activity; AED: antiepileptic drug; CSF: cerebrospinal fluid; EEG: electroencephalography; IVIG: intravenous immunoglobulin; Mass lesion: a composite variable comprising ischemic infarct, intracranial hemorrhage, or cerebral edema; PLEX: plasmapheresis; SD: standard deviation

Table 1: Distribution of various variables by COVID severity, status epilepticus, and cortical myoclonic activity

\section{Clinical characteristics}

The mean age of patients was 47.8 years (SD 25.6), with males comprising 99 (56.3\%) patients. Severe COVID-19 was seen in 90 (53.6\%) patients. The average LOS was 22.1 days (SD 15.3). Patients with severe COVID-19 and CMA had prolonged LOS, but status epilepticus patients did not. The average GCS was 10.4 (SD 4.3). Of those with severe disease, 23 (46.9\%) had status epilepticus and 25 (67.6\%) had CMA (Table 1). Patients with severe COVID-19 were older and had lower GCS. Patients with CMA were also older on average. Seventy-four (51.0\%) patients required intubation at some point during admission, with greater proportion seen in severe COVID-19, status epilepticus, and CMA. Two multivariate regression models were created to assess predictors of status epilepticus and CMA (Table 2). The only significant predictor for status epilepticus was intracranial mass lesion (a composite variable comprising infarct, ICH, or edema). For CMA, the significant predictive variables were severe COVID-19, age, and male sex.

\section{Workup and diagnosis}

EEG findings were reported for 103 patients. Seizure-like abnormality on EEG was found in 52 (50.5\%) patients, of which 30 (57.7\%) had electrographic seizures and $16(30.8 \%)$ had electrographic status. Patients with status epilepticus had more EEG abnormalities whereas patients with CMA had fewer (Table 1). Moderate/severe generalized slowing was reported in 66 (71.0\%) patients. Severe COVID-19 was not associated with electrographic seizure-like activity or generalized slowing. Patients with status epilepticus had more seizure-like activity and generalized slowing recorded. 
CSF analysis was reported for $92(52.3 \%)$ patients, of which, 40 (43.5\%) patients had abnormal CSF findings. CSF cultures were negative in all cases. COVID-19 PCR was positive in the CSF from 6 patients, with 1 (1.1\%) having elevated IgM and IgG antibodies against COVID-19. Severe COVID-19, status epilepticus, and CMA were not associated with abnormal CSF.

An underlying neurologic diagnosis was discovered for 91 (51.7\%) patients, of which 47 (51.6\%) had encephalitis, 18 (19.8\%) had cerebral infarct, and 14 (15.4\%) had intracranial hemorrhage (ICH) (Figure 2). Uncommon diagnoses included edema (posterior reversible encephalopathy syndrome), microhemorrhages, and venous thrombosis. A secondary neurological diagnosis was discovered in 17 (18.7\%) of these patients, of which 7 had venous sinus thrombosis, 4 had cerebral microhemorrhages, 3 had ICH, 2 had cerebral edema, and 1 had a brain tumor (Figure 2). The remaining 85 (48.3\%) patients had an unknown, if any, underlying neurologic diagnosis. A diagnosis of encephalitis was associated with a trend towards less severe COVID-19 infection ( $\mathrm{p}=0.089$ ) (Table 1). A distinction between encephalitis resulting directly from COVID-19 or from an autoimmune response could not be made. Encephalitis was not associated with CMA or status epilepticus.

\section{Distribution of primary underlying neurologic diagnosis}

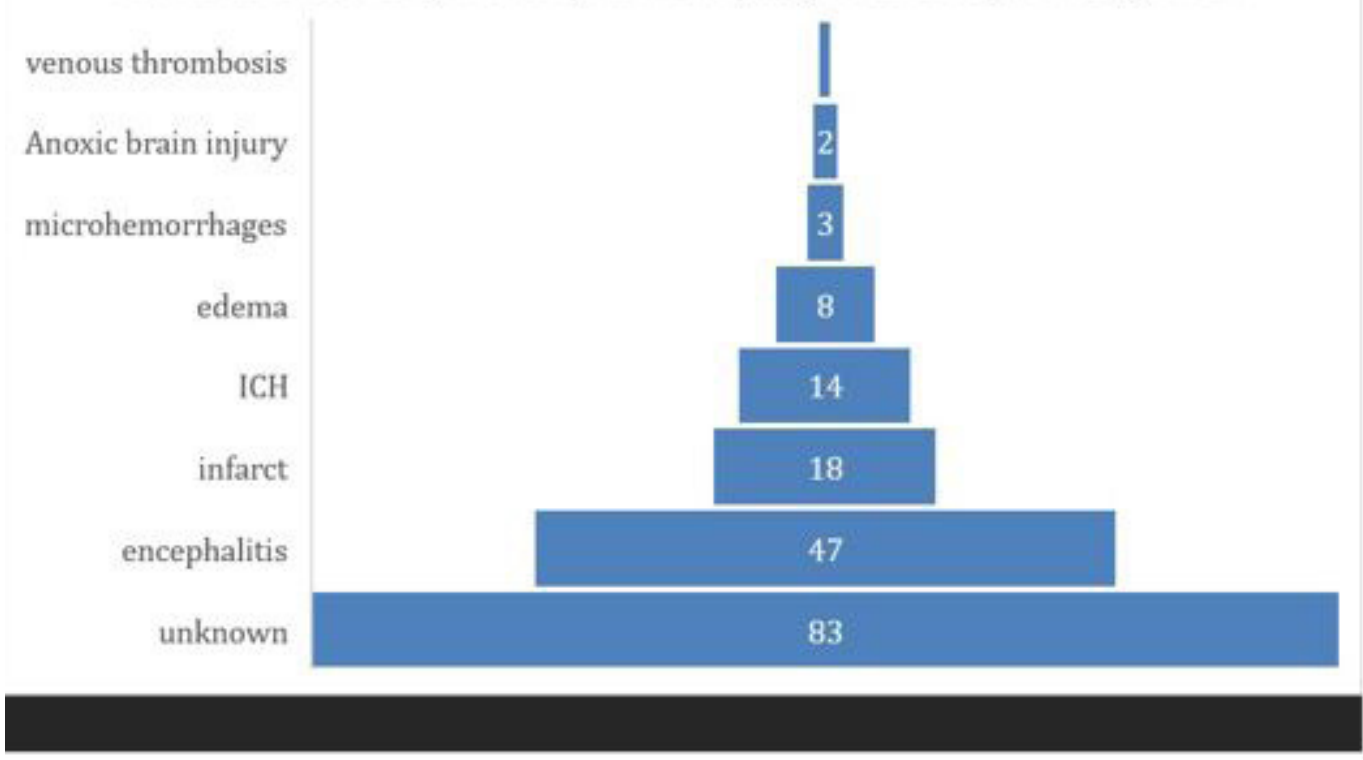

Distribution of secondary underlying neurologic diagnosis

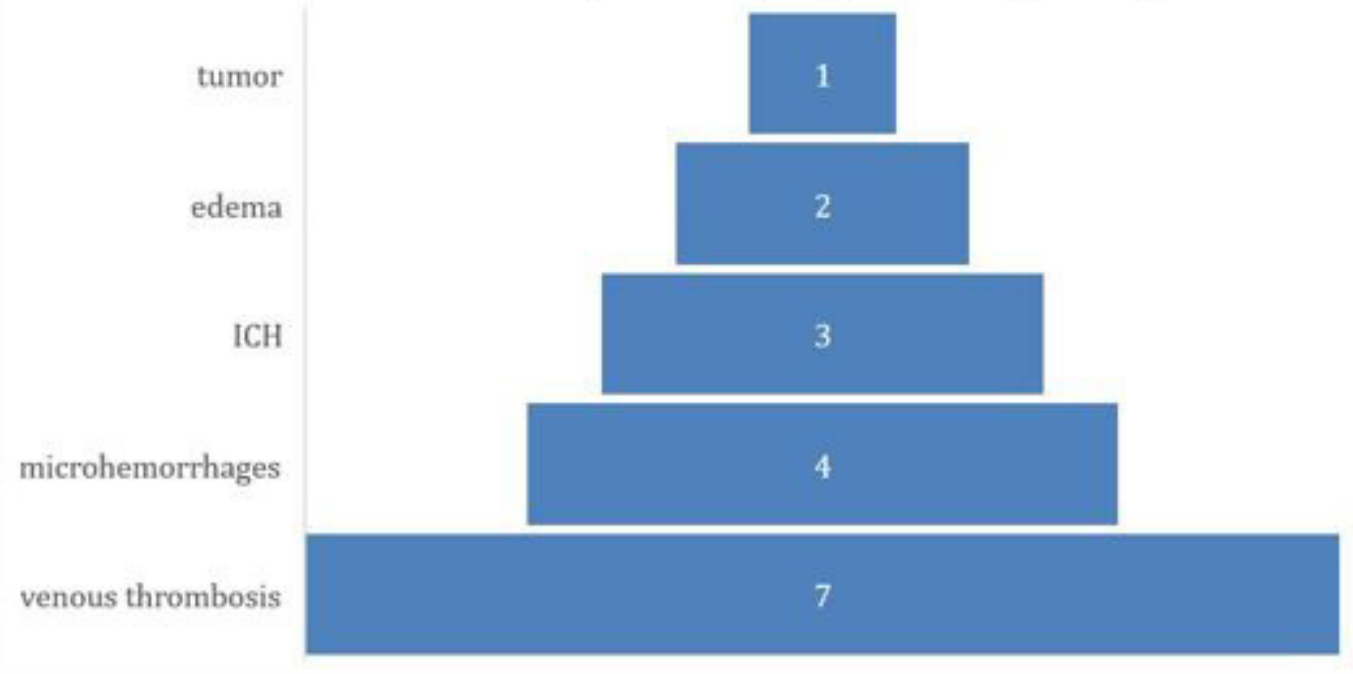

Figure 2: Distribution of primary and secondary neurologic diagnosis Edema implies cerebral edema resulting from posterior reversible encephalopathy syndrome. (ICH - intracranial hemorrhage) 


\begin{tabular}{|c|c|c|c|c|c|c|}
\hline Variable & $\begin{array}{c}\text { Odds ratio for good } \\
\text { outcome }\end{array}$ & $\mathbf{9 5 \%}$ CI & p-value & $\begin{array}{c}\text { Odds ratio for } \\
\text { mortality }\end{array}$ & 95\% CI & p-value \\
\hline Severe COVID-19 & 0.116 & $0.043-0.315$ & $<0.001$ & 8.307 & $1.964-35.145$ & 0.004 \\
\hline Age & 0.980 & $0.962-0.999$ & 0.038 & 1.022 & $0.999-1.046$ & 0.064 \\
\hline Male sex & 1.325 & $0.555-3.162$ & 0.526 & 0.652 & $0.235-1.809$ & 0.411 \\
\hline Status epilepticus & 0.651 & $0.255-1.660$ & 0.369 & 1.461 & $0.509-4.200$ & 0.481 \\
\hline Cortical myoclonic & 1.402 & $0.469-4.190$ & 0.545 & 0.375 & $0.103-1.365$ \\
\hline activity & 0.303 & $0.107-0.856$ & 0.024 & 2.208 & 0.137 \\
\hline Intubation & 26.668 & $6.337-112.223$ & $<0.001$ & 0.012 & $0.002-0.086$ \\
\hline Coefficient & $0.656-8.777$ & 0.261 \\
\hline
\end{tabular}

Table 2: Multivariate regression models assessing the associations with various covariates, for good outcome and mortality

\section{Treatment \& management}

Antiepileptic drug (AED) treatment was reported in 133 (75.6\%) patients. The most frequently utilized AEDs were levetiracetam (71.5\%) and valproate (19.2\%). The mean number of AEDs was 1.4 (SD 1.0), with more AEDs used in treatment of status than other patients. For immunomodulatory treatments, 32 (19.3\%) patients were treated with pulse dose steroids, 22 (13.4\%) received IVIG, and 4 (2.4\%) received PLEX. More pulse dose steroids and IVIG were used with a diagnosis of encephalitis. Overall, steroids of any dose were used in 41 patients (34.5\%), with a greater proportion used in severe COVID-19, status epilepticus, and CMA. Remdesivir was used in 8 patients $(8.0 \%)$ and tocilizumab was used in $4(4.1 \%)$.

\section{Outcomes}

Overall, 101 (63.9\%) patients experienced a good outcome, while only 24 (14.6\%) died. Patients with severe COVID-19 and mass lesion had less good outcomes, whereas those with encephalitis had more good outcomes. Similar findings were observed with mortality, except that there was a non-significant trend towards more mortality with status epilepticus.

In order to determine the association between good outcomes and various explanatory variables, a multi-variate regression model was created with good outcome as the dependent variable, and COVID-19 severity, age, sex, status epilepticus, CMA, and intubation as explanatory variables (Table 3). The model explained $31.9 \%$ of the variability in the outcome. Severe COVID-19 infection (OR $=$ $0.116, \mathrm{p}<0.001)$, increasing age $(\mathrm{OR}=0.980, \mathrm{p}=0.038)$ and intubation $(\mathrm{OR}=0.303, \mathrm{p}=0.024)$ were associated with reduced odds of good outcome. For mortality, similar covariates were used and the model explained $23.1 \%$ of variability in the outcome. Only severe COVID-19 was associated with higher mortality $(\mathrm{OR}=8.31 ; \mathrm{p}=0.004)$.

As encephalitis was associated with good outcomes on univariate analysis, an additional multivariate regression model was constructed, with good outcome as the dependent variable and encephalitis, age, sex, IVIG, pulse steroids, and severe COVID-19 as covariates (Table 4). The model explained $23.9 \%$ of variability in the outcome. Encephalitis was associated with higher odds of good outcome $(\mathrm{OR}=6.07 ; \mathrm{p}=0.004)$, while severe COVID-19 remained associated with lower odds $(\mathrm{OR}=0.19 ; \mathrm{p}=0.002)$. For mortality with 
similar covariates, encephalitis had a non-significant trend towards lower mortality $(\mathrm{OR}=0.20, \mathrm{p}=0.067)$ whereas severe COVID-19 was no longer associated with mortality. Male sex also had a non-significant trend towards lower mortality, whereas pulse steroids had a non-significant trend towards higher mortality.

\begin{tabular}{|c|c|c|c|c|c|c|}
\hline Variable & $\begin{array}{c}\text { OR for status } \\
\text { epilepticus }\end{array}$ & $\mathbf{9 5 \%}$ CI & p-value & $\begin{array}{c}\text { OR for cortical } \\
\text { myoclonic activity }\end{array}$ & $\mathbf{9 5 \%}$ CI & $\mathbf{p}$-value \\
\hline Severe COVID-19 & 0.632 & $0.191-2.094$ & 0.453 & 8.636 & $1.547-48.204$ & 0.014 \\
\hline Age & 1.008 & $0.983-1.034$ & 0.539 & 1.047 & $1.004-1.091$ & 0.030 \\
\hline Male sex & 0.946 & $0.294-3.042$ & 0.925 & 8.124 & $1.393-47.388$ & 0.020 \\
\hline Abnormal CSF & 2.183 & $0.541-8.808$ & 0.925 & 1.397 & $0.256-7.635$ \\
\hline Mass lesion & 4.723 & $1.054-21.165$ & 0.273 & 0.700 \\
\hline Coefficient & 0.195 & $0.021-1.783$ & 0.148 & 0.003 & $0.032-1.967$ \\
\hline
\end{tabular}

CI: confidence interval; CSF: cerebrospinal fluid; OR: odd's ratio

Table 3: Multivariate regression models assessing the associations with various covariates. for status epilepticus and cortical myoclonic activity

\begin{tabular}{|c|c|c|c|c|c|c|}
\hline Variable & $\begin{array}{c}\text { OR for good } \\
\text { outcome }\end{array}$ & 95\% CI & p-value & OR for mortality & 95\% CI & p-value \\
\hline Encephalitis & 6.066 & $1.756-20.949$ & 0.004 & 0.200 & $0.036-1.123$ & 0.067 \\
\hline Age & 0.985 & $0.961-1.010$ & 0.244 & 1.013 & $0.977-1.051$ & 0.477 \\
\hline Male sex & 2.194 & $0.734-6.556$ & 0.159 & 0.239 & $0.053-1.077$ & 0.062 \\
\hline IVIG & 0.421 & $0.095-1.874$ & 0.256 & 0.637 & $0.083-4.918$ & 0.666 \\
\hline Pulse steroids & 0.690 & $0.188-2.533$ & 0.576 & 4.125 & $0.773-22.006$ & 0.091 \\
\hline Severe COVID-19 & 0.186 & $0.064-0.543$ & 0.002 & 2.816 & $0.605-13.115$ & 0.187 \\
\hline Coefficient & 2.712 & $0.552-13.320$ & 0.219 & 0.107 & $0.012-0.990$ & 0.049 \\
\hline
\end{tabular}

CI: confidence interval; IVIG: intravenous immunoglobulin; OR: odd's ratio

Table 4: Multivariate regression models assessing the associations with encephalitis as main explanatory variable, for good outcomes and mortality 


\section{Discussion}

In the present study, the clinical characteristics, underlying etiologies, treatments, and outcomes of 176 patients presenting with seizures and/or Cortical Myoclonic Activity (CMA) and COVID-19 infection are described, with individual patient data aggregated from various case series and case reports. The majority of patients had new-onset seizures. A significant proportion of patients had good outcomes. Despite the severity of COVID-19 infection being the major determinant of poor outcomes, encephalitis as an underlying etiology was independently predictive of good outcomes. Presence of status epilepticus and CMA did not worsen clinical outcomes. Where diagnosis was apparent, patients were appropriately and promptly managed using standard seizure and/or immunomodulatory treatments. This highlights the efficacy of existing treatment protocols. The diagnosis of encephalitis was based either on findings of: [1] characteristic inflammatory changes on MR imaging, which reversed in some cases [2,17], rapid reversal of neurologic disability with immunomodulatory treatments [3,18], isolation of COVID-19 nucleic acid from CSF [8]. CSF analysis was also pertinent in its diagnosis, and may be normal [19], demonstrate pleocytosis, or show elevated pro-inflammatory cytokines. Specifically, elevated levels of IL-6, IL-8, IL-10, and TNF-a have been reported [20]. These may indirectly implicate neuroinvasiveness of COVID-19 and indeed in an autopsy study, over 50\% of brain specimens had COVID-19 detected with brainstem and cerebellar neuroinflammation [21]. However, isolating the virus from CSF has been notoriously difficult, and indirect methods such asincreased CSF IgM for COVID-19 S1 protein may be a better reflection of CNS infection [18,20,22]. This protein itself can cross through the blood brain barrier and is taken up by multiple brain regions.

Other instances of encephalitis may be due to autoimmune phenomena, whereby exposure or modification of intracellular antigens due to severe systemic inflammatory response and molecular mimicry from viral particles can lead to autoantibody production [23]. Previous research has shown elevated serum and CSF anti-neuronal and anti-glial antibodies in COVID-19 patients with neurologic symptoms [7]. One of the antigens was the NMDA receptor, and the patient who had co-existence of COVID-19 virus in CSF showed anti-NMDA IgG antibodies [7,24]. Despite severe neurologic symptoms, the patient was discharged with full recovery after treatment with methylprednisolone and IVIG. Similar response to immunotherapy has been reported elsewhere [25,26]. In our study, outcomes were much improved with identification of encephalitis as the primary etiology, thereby highlighting both the efficacy of targeted treatments, and the importance of complete neurologic workup.

Overall, most patients achieved good outcomes even in the presence of status epilepticus and CMA, demonstrating that current antiepileptic treatment protocols work. Outcomes were predominantly determined by the severity of COVID-19 infection and diagnosis of encephalitis in multivariate models. Unsurprisingly, higher age and intubation status were also independently associated with poor outcomes. However, no such association existed with status epilepticus or CMA. Some patients with status epilepticus had prolonged LOS and still achieved good outcomes, which agrees with findings from the literature that LOS is not associated with outcomes or severity of COVID-19 [27]. No sex discrepancy for outcomes was noted, except for a higher propensity for CMA, despite a global meta-analysis identifying male sex as a significant risk factor for mortality [28]. This may be due to a predominance of male patients in the sample and the nature of the study, although sex disparities are a crucial frontier to investigate further in COVID-19. Most patients were treated with AEDs, and levetiracetam was the most common, which is likely due to its favorable side effect profile and lower propensity for drug-interactions. Immunomodulatory treatments, except for steroids used specifically for COVID-19, were not widely used, which is likely attributable to the large proportion of cases with unknown etiology.

Untreated status epilepticus significantly worsens outcomes, so it is critical to use EEG to monitor patients. EEG abnormalities have been previously highlighted in COVID-19 within various systematic reviews and retrospective cohorts [6,29-32], with diffuse slowing being most common followed by epileptiform abnormalities. The latter could be secondary to cortical irritability from possible viral neuroinvasion, sepsis from COVID-19, or presence of other neurologic comorbidities. An interesting observation reported in the literature is the presence of frontal epileptiform discharges, which have been correlated with viral neuroinvasion through naso-oral mucosa via ACE-2 receptors and subsequent translocation through olfactory channels into the orbitofrontal cortex [33-36]. These specific EEG abnormalities were not identified in the present review's cohort, but nevertheless their identification is crucial. 
CMA was noted in $24 \%$ of patients and was generalized in nature. In the included studies, CMA was accompanied by significant mental status changes, and in most cases, treatment with antiepileptics was pursued [2,3,37]. CMA is a marker of severe cerebral dysfunction either perpetuated by epileptogenicity, direct neuronal damage from traumatic or hypoxic injury, severe systemic metabolic disturbances, or an immune-mediated mechanism. This is evidenced by a greater proportion of CMA in severe COVID-19 infection, where all of these mechanisms may be involved, but especially hypoxia, even if it is subclinical in nature [38]. The same mechanisms could be involved in precipitating seizures as well. Treatment response is dependent on the etiology. Similar to the present study, other authors have not noted a detrimental impact on outcomes with CMA in COVID-19, but have noted at least partial response to immunotherapy [37,39]. Future studies should investigate whether the underlying autoimmune mechanisms respond to immunomodulatory treatments or are self-limiting [37].

\section{Limitations}

Due to this study's reliance on case reports and case series, we are unable to comment on the overall incidence of seizures or CMA in COVID-19. Similarly, we could not perform a meta-analysis as there is significant heterogeneity in the studies investigated. Long-term follow-up was sparingly reported, but where available, it was used to designate outcomes. It would be interesting for future studies to investigate whether COVID-19 is associated with the development of epilepsy, seizure recurrence, or cognitive dysfunction. The COVID long-haul, otherwise also known as chronic COVID syndrome, has been linked to the development of multi-organ manifestations including that of CNS.(40) Understanding the impact and long-term outcomes of patients with either new-onset or pre-existing seizures should be considered an important research priority.

\section{Conclusion}

Most patients achieved good outcomes, regardless of the presence of status epilepticus or Cortical Myoclonic Activity (CMA). Despite severe COVID-19 strongly predicting poor outcomes, identification of encephalitis as an underlying etiology was independently associated with good outcomes. Therefore, obtaining a comprehensive neurologic workup to identify an underlying etiology of seizures and CMA is strongly recommended to direct targeted antiepileptic and immunomodulatory treatments. Further research should focus on elucidating the pathophysiology of encephalitis in COVID-19, and investigate long-term neurologic outcomes 


\section{References}

1. World Health Organization (WHO) (2021) Coronavirus Disease (COVID-19), Geneva, Switzerland.

2. Emanuela K, Giovanna B, Sebastian W, Imbach Lukas L, Daniel K, et al. (2020) Large and Small Cerebral Vessel Involvement in Severe COVID-19. Stroke 51: 3719-22.

3. Beach SR, Praschan NC, Hogan C, Dotson S, Merideth F, et al. (2020) Delirium in COVID-19: A case series and exploration of potential mechanisms for central nervous system involvement. Gen Hosp Psychiatry 65: 47-53.

4. Radmard S, Epstein SE, Roeder HJ, Michalak AJ, Shapiro SD, et al. Inpatient Neurology Consultations During the Onset of the SARS-CoV-2 New York City Pandemic: A Single Center Case Series. Front Neurol. 2020;11:805.

5. Kurd M, Hashavya S, Benenson S, Gilboa T. Seizures as the main presenting manifestation of acute SARS-CoV-2 infection in children. Seizure. 2021 Nov;92:89-93.

6. Waters BL, Michalak AJ, Brigham D, Thakur KT, Boehme A, et al. (2021) Incidence of Electrographic Seizures in Patients With COVID-19. Front Neurol 12: 614719.

7. Franke C, Ferse C, Kreye J, Reincke SM, Sanchez-Sendin E, et al. (2021) High frequency of cerebrospinal fluid autoantibodies in COVID-19 patients with neurological symptoms. Brain Behav Immun 93: 415-9.

8. Rezaeitalab F, Jamehdar SA, Sepehrinezhad A, Rashidnezhad A, Moradi F, et al. (2021) Detection of SARS-coronavirus-2 in the central nervous system of patients with severe acute respiratory syndrome and seizures. J Neurovirol 1: 1-6.

9. Mao L, Jin H, Wang M, Hu Y, Chen S, et al. (2020) Neurologic Manifestations of Hospitalized Patients With Coronavirus Disease 2019 in Wuhan, China. JAMA Neurol 77: 683-90.

10. Frontera JA, Sabadia S, Lalchan R, Fang T, Flusty B, et al. (2021) A Prospective Study of Neurologic Disorders in Hospitalized Patients With COVID-19 in New York City. Neurology 96: e575-86.

11. Romero-Sánchez CM, Díaz-Maroto I, Fernández-Díaz E, Sánchez-Larsen Á, Layos-Romero A, et al. (2020) Neurologic manifestations in hospitalized patients with COVID-19: The ALBACOVID registry. Neurology e1060-70.

12. Lu L, Xiong W, Liu D, Liu J, Yang D, et al. (2020) New onset acute symptomatic seizure and risk factors in coronavirus disease 2019: A retrospective multicenter study. Epilepsia 61: e49-53.

13. Sandoval F, Julio K, Méndez G, Valderas C, Echeverría AC, et al. (2021) Neurologic Features Associated With SARS-CoV-2 Infection in Children: A Case Series Report. J Child Neurol 36: 853-66.

14. Liberati A, Altman DG, Tetzlaff J, Mulrow C, Gøtzsche PC, et al. (2009) The PRISMA statement for reporting systematic reviews and meta-analyses of studies that evaluate healthcare interventions: explanation and elaboration. BMJ 339: b2700.

15. Bramer WM, de Jonge GB, Rethlefsen ML, Mast F, Kleijnen J (2018) A systematic approach to searching: an efficient and complete method to develop literature searches. J Med Libr Assoc JMLA 106: 531-41. 
16. NHLBI, NIH (2021) Study Quality Assessment Tools, USA.

17. Manzo ML, Galati C, Gallo C, Santangelo G, Marino A, et al. (2021) ADEM post-Sars-CoV-2 infection in a pediatric patient with Fisher-Evans syndrome. Neurol Sci 12: 1-4.

18. Gunawardhana C, Nanayakkara G, Gamage D, Withanage I, Bandara M, et al. (2021) Delayed presentation of postinfectious encephalitis associated with SARS-CoV-2 infection: a case report. Neurol Sci 17: 1-4.

19. Helms J, Kremer S, Merdji H, Clere-Jehl R, Schenck M, et al. (2020) Neurologic Features in Severe SARS-CoV-2 Infection. N Engl J Med 382: 2268-70.

20. Benameur K, Agarwal A, Auld SC, Butters MP, Webster AS, et al. (2020) Encephalopathy and Encephalitis Associated with Cerebrospinal Fluid Cytokine Alterations and Coronavirus Disease, Atlanta, Georgia, USA, 2020. Emerg Infect Dis 26: $2016-21$.

21. Matschke J, Lütgehetmann M, Hagel C, Sperhake JP, Schröder AS, et al. (2020) Neuropathology of patients with COVID-19 in Germany: a post-mortem case series. Lancet Neurol 19: 919-29.

22. Rhea EM, Logsdon AF, Hansen KM, Williams LM, Reed MJ, et al. (2020) The S1 protein of SARS-CoV-2 crosses the blood-brain barrier in mice. Nat Neurosci 16: 1-11.

23. Suurmond J, Diamond B (2015) Autoantibodies in systemic autoimmune diseases: specificity and pathogenicity. J Clin Invest 125: 2194-202.

24. Allahyari F, Hosseinzadeh R, Nejad JH, Heiat M, Ranjbar R (2021) A case report of simultaneous autoimmune and COVID-19 encephalitis. J Neurovirol 26: 1-3.

25. Cao A, Rohaut B, Le Guennec L, Saheb S, Marois C, et al. (2020) Severe COVID-19-related encephalitis can respond to immunotherapy. Brain 143: e102-e102.

26. Paterson RW, Brown RL, Benjamin L, Nortley R, Wiethoff S, et al. (2020) The emerging spectrum of COVID-19 neurology: clinical, radiological and laboratory findings. Brain 143: 3104-20.

27. Monti G, Giovannini G, Marudi A, Bedin R, Melegari A, et al. (2020) Anti-NMDA receptor encephalitis presenting as new onset refractory status epilepticus in COVID-19. Seizure 81: 18-20.

28. Peckham H, de Gruijter NM, Raine C, Radziszewska A, Ciurtin C, et al. (2020) Male sex identified by global COVID-19 metaanalysis as a risk factor for death and ITU admission. Nat Commun 11: 6317.

29. Lin L, Al-Faraj A, Ayub N, Bravo P, Das S, et al. (2021) Electroencephalographic Abnormalities are Common in COVID-19 and are Associated with Outcomes. Ann Neurol 89: 872-83.

30. Antony AR, Haneef Z (2020) Systematic review of EEG findings in 617 patients diagnosed with COVID-19. Seizure - Eur J Epilepsy 83: 234-41.

31. Petrescu A-M, Taussig D, Bouilleret V (2020) Electroencephalogram (EEG) in COVID-19: A systematic retrospective study. Neurophysiol Clin 50: 155-65. 
32. Danoun OA, Zillgitt A, Hill C, Zutshi D, Harris D, et al. (2021) Outcomes of seizures, status epilepticus, and EEG findings in critically ill patient with COVID-19. Epilepsy Behav 118: 107923.

33. De Stefano P, Nencha U, De Stefano L, Mégevand P, Seeck M (2020) Focal EEG changes indicating critical illness associated cerebral microbleeds in a Covid-19 patient. Clin Neurophysiol Pract 5: 125-9.

34. Guennec LL, Devianne J, Jalin L, Cao A, Galanaud D, et al. (2020) Orbitofrontal involvement in a neuro COVID-19 patient. Epilepsia 61: e90-4.

35. Galanopoulou AS, Ferastraoaru V, Correa DJ, Cherian K, Duberstein S, et al. (2020) EEG findings in acutely ill patients investigated for SARS-CoV-2/COVID-19: A small case series preliminary report. Epilepsia Open 5: 314-24.

36. DosSantos MF, Devalle S, Aran V, Capra D, Roque NR, et al. (2020) Neuromechanisms of SARS-CoV-2: A Review. Front Neuroanat 14: 37.

37. Rábano-Suárez P, Bermejo-Guerrero L, Méndez-Guerrero A, Parra-Serrano J, Toledo-Alfocea D, et al. (2020) Generalized myoclonus in COVID-19. Neurology 95: e767-72.

38. Cascella M, Rajnik M, Cuomo A, Dulebohn SC, Di Napoli R (2022) Features, Evaluation, and Treatment of Coronavirus. 2022 Jan 5. In: StatPearls [Internet]. Treasure Island (FL): StatPearls Publishing, USA.

39. Anand P, Zakaria A, Benameur K, Ong C, Putman M, et al. (2020) Myoclonus in Patients With Coronavirus Disease 2019: A Multicenter Case Series. Crit Care Med 48: 1664-9.

40. Baig AM (2020) Deleterious Outcomes in Long-Hauler COVID-19: The Effects of SARS-CoV-2 on the CNS in Chronic COVID Syndrome. ACS Chem Neurosci 11: 4017-20. 